\title{
Edge Detection in Satellite Image Using Cellular Neural Network
}

\author{
Osama Basil Gazi(*) \\ Faculty of computers and \\ information. Helwan University \\ 10 Yahya Shaheen St., El-Taween, \\ El-Haram, Giza
}

\author{
Dr. Mohamed Belal \\ Faculty of Computers and \\ Information \\ Helwan University
}

\author{
Dr. Hala Abdel-Galil \\ Faculty of Computers and \\ Information \\ Helwan University
}

\begin{abstract}
The present paper proposes a novel approach for edge detection in satellite images based on cellular neural networks. CNN based edge detector in used conjunction with image enhancement and noise removal techniques, in order to deliver accurate edge detection results, compared with state of the art approaches. Thus, considering the obtained results, a comparison with optimal Canny edge detector is performed. The proposed image processing chain deliver more details regarding edges than canny edge detector. The proposed method aims to preserve salient information, due to its importance in all satellite image processing applications.
\end{abstract}

Keywords-cellular neural network; liner matrix inequality; differential evolution; genetic algorithm; Field-programmable gate array; partial differential equation

\section{INTRODUCTION}

The use of satellite imagery in everyday life is no more a novelty. The first satellite for acquiring land areas imaging was placed in orbit in 1972 (Landsats) (1). The rapid advances and accessibility of computer technology brought satellite images in millions of homes, cars, schools, and offices. Satellite imagery provides accurate information of observing and quantifying the surface of the earth. The main benefit is the increased knowledge about our environment.

\section{A. Satellite imagery}

Satellite image acquisition looks at the Earth differently. It has no camera, but its instruments are "sensitive" to visible light, but also to other parts of 'electromagnetic spectrum' as the infrared, ultraviolet or microwaves. These instruments (scanners) scan the surface of the Earth and record measures 'light' which are then used by computer programs for create images. The stages of acquiring the satellite images are as follows. The radiation emitted by an energy source or illumination (A) covers a distance and interacts with the atmosphere (B) before reaching the target (C). The energy interacts with the surface of the target, depending on the characteristics of and radiation properties of the surface. Radiation is reflected or scattered to the sensor (D), which registers and then can transmit the energy by remote means to a receiving station $(\mathrm{E})$ where information is transformed into images (digital or photographic). A visual interpretation of digital the image $(\mathrm{F})$ is then required to extract the information that is desired on target. The final step is to use the information extracted from the image to better understand the target for us to discover new aspects or to help solve a particular problem.
There are many applications of satellite images in fields such as meteorology, agriculture, geology, forestry, landscape, biodiversity conservation, regional planning, education, intelligence and warfare. Images can be in visible colors and in other spectra. Satellite imagery has two main elements:

- Spatial information, described by the pixel size of the imagery and,

- Spectral information.

We can only see a small proportion of the electromagnetic spectrum. Satellite sensors pick up information in a much wider range allowing us to look at the infrared, thermal and microwave signatures being returned from the earth's surface. Looking at information from these bands allows us to pick up patterns and relationships we would previously not have seen. This is the real power of remote sensing imagery. The satellite senses electromagnetic energy at different wavelengths. Examples of imagery from three wavelengths most commonly shown on weather broadcasts are mentioned: visible images, infrared imagery and water vapor imagery.

Regarding satellite images in remote sensing, there are four types of resolutions: spatial, spectral, temporal, and radiometric. Campbell (3) defines these as follows: spatial resolution represents the pixel size of an image of the surface area measured on the ground, determined by the sensor's instantaneous field of view, spectral resolution is defined by the wavelength interval size within a segment of the Electromagnetic Spectrum and number intervals that the sensor is measuring, temporal resolution is defined by the amount of time between two consequent image acquisitions for a given surface location, radiometric resolution is defined as the ability of an imaging system to record many levels of brightness (contrast for example). This actually defines the bit-depth of the sensor (number of grayscale levels) and is typically expressed as 8-bit (0-255), 11-bit (0-2047), 12-bit (0-4095) or 16-bit $(0-65,535)$

\section{1) Application of satellite imagery}

Thus, within satellite images, salient information describes different areas, also objects within images, which are of high importance in applications in fields like meteorology, agriculture, geology, forestry and many others. Satellite and aerial images on the Internet have many useful commercial applications. Farmers use it to monitor crops for blight and other problems and to deploy localized remedies when needed. Land use managers use it to assess and plan city growth. 
Insurance companies use before-and-after imagery to verify damage claims after floods, hurricanes, and other disasters. The media routinely adds satellite imagery to news reports to illustrate where important events have occurred. Software developers incorporate satellite imagery into flight simulators, games, and even wireless handheld devices. Satellite imagery is most useful when combined with GPS, electronic maps, and localized data into a geographic information system. Perhaps the most popular example of this is the Google Earth application, which recently made commercial satellite imagery freely available to almost anyone on the planet via the Internet. Some of the industries that can potentially benefit from Google Earth Pro, a premium-paid service on Google Earth, include commercial real estate, residential real estate, architecture/engineering, insurance, media, defense/intelligence, homeland security, public sector, and state and local government.

\section{B. Cellular Neural Networks}

There are two types of vision systems, artificial and natural vision systems, the latter one being characterized by continuous time and signal values as opposed to the first one. In particular for the natural vision systems, the cells of the natural retina combine photo transduction and collective parallel processing for the realization of low-level image processing operations (feature extraction, motion analysis, etc.), concurrently with the acquisition of the image. Thus, spatial representation of the spatial-temporal representation exists. The Cellular Neural Networks (CNN) are considered as a unifying model for spatio-temporal properties of the visual system. $(8,9)$

Cellular Neural Networks (CNN) and the CNN universal machine (CNN-UM) were introduced in 1988 and 1992, respectively $[8,9]$. The definition of such networks is that they are arrays of identical dynamical systems, called cells, that are locally connected [9]. Each cell, the basic unit of a CNN, is a one-dimensional dynamic system connected only to its neighbor cells, i.e. adjacent cells interact directly with each other Figure 13. Cells within the close vicinity have indirect effect because of the propagation effects of the dynamics in the network. The cell located in the position $(i, j)$ of a twodimensional $M \times N$ array is denoted by $C_{i j}$, and its $r$ neighborhood $N^{r}{ }_{i j}$ is defined by. (1)

$$
N^{r}{ }_{i j}=\left\{C_{k l} \mid \max \{|k-i|,|l-j|\} \leq r, 1 \leq k \leq M, 1 \leq l \leq N\right\}
$$

The basic circuit unit of a CNN is called a cell. It contains linear and nonlinear elements, which typically act like linear capacitors, linear resistors, linear and nonlinear controlled sources, and independent sources. The main characteristic of each cell of the neural network are: a constant external input $u$, and an output $y$. The equivalent block diagram of each neural network cell is as shown in the figure 2 .

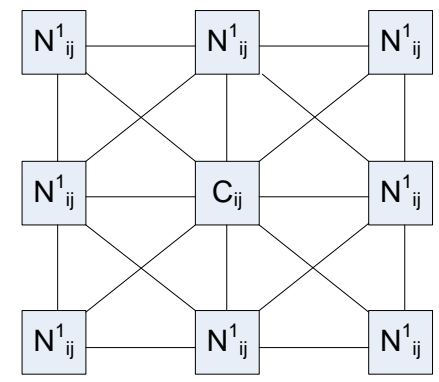

Fig. 1. CNN; the cell $C i j$ with its neighborhood for $r=1$;

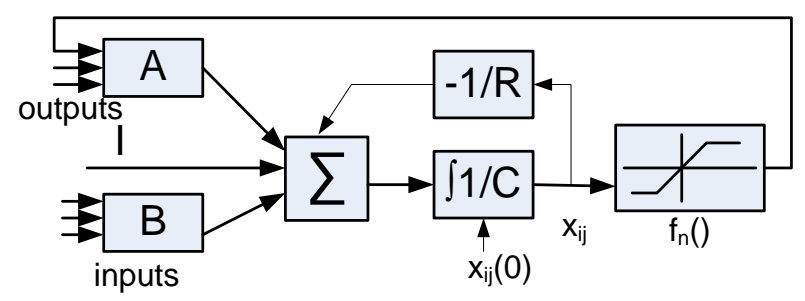

Fig. 2. Block diagram of a cell;

The first-order non-linear differential equation defining the dynamics of a cellular neural network can be written as follows:

$$
\begin{aligned}
& C \frac{\partial x_{i j}(t)}{\partial t}=-\frac{1}{R} x_{i j}(t)+\sum_{C_{k l} \in N^{r} i j} A(i, j ; k, l) y_{k l}(t)+\ldots \\
& \ldots+\sum_{C_{k l} \in N^{r}{ }_{i j}} B(i, j ; k, l) u_{k l}(t)+I
\end{aligned}
$$

Where

- $x_{i j}$ is the state of cell $C_{i j}$;

- $\mathrm{C}$ and $\mathrm{R}$ represent the integration time constant of the system;

- $\quad I$ is an independent bias constant;

- $y_{i j}(t)=f\left(x_{i j}(t)\right)$, where $f$ is a chosen function a commonly used $f$ function is:

$$
f(x)=\frac{1}{2}(|x+1|-|x-1|\}
$$

The matrices $\mathrm{A}($.$) and \mathrm{B}($.$) are known as cloning templates.$

- $\mathrm{A}($.$) acts on the output of neighbouring cells and is$ referred to as the feedback operator;

- $\mathrm{B}($.$) in turn affects the input control and is referred to$ as the control operator. 
$\mathrm{A}($.$) and \mathrm{B}($.$) depend on the application. In image$ processing, were an image is a rectangular array having $N$ and $M$ are the numbers of rows and columns, respectively. Each cell in a CNN corresponds to an element of the array. Assuming that each cell is connected to its nearest neighbors only ("3*3-neighborhood") and that the local connections of a cell do not depend on the cell's position, the Template set contains 19 coefficients (A-Template: a1 .. a9, B-Template: b1 .. b9, Bias I). The behavior of the CNN is completely determined by this Template set. This will be the approach to be further on used in the CNN implementation.

The most popular application for $\mathrm{CNN}$ has been in image processing, essentially because of their analog feature and sparse connections, which are conductive to real time processing (8), (10). A two dimensional CNN can be viewed as a parallel non-linear two-dimensional filter and, calibrating the A, B, I parameters can be used for noise removal (11), shape extraction, edge detection, inpainting (12).

There are two types of CNN: continuous time (CT-CNN) and discrete time (DT-CNN) cellular neural networks. Considering the discrete nature of any kind of image information, the second one will be used in implementations involving image processing algorithms.

\section{Continuous CNN}

They are described by equation (3). It contains continuous linear and nonlinear circuit elements, which typically are linear capacitors, linear resistors, linear and nonlinear controlled sources, and independent sources. All the cells of a CNN have the same circuit structure and element values. A continuous typical circuit of a single cell is shown in the figure below (13).

Each cell contains one independent voltage source $E^{u}{ }_{i j}$ (Input), one independent current source I (Bias), several voltage controlled current sources $I_{n}{ }^{u}{ }^{i j}, I_{n}{ }^{y}{ }^{i j}$, and one voltage controlled voltage source $E_{y i j}$ (Output). The controlled current sources $I_{n}{ }^{u i j}$ are coupled to neighbor cells via the control input voltage of each neighbor cell. Similarly, the controlled current sources $I_{n}{ }^{y i j}$ are coupled to their neighbor cells via the feedback from the output voltage of each neighbor cell.

The cell $C_{(i, j)}$ has direct connections to its neighbors through two kinds of weights: the feedback weights $a(k, l ; i, j)$ and the control weights $b(k, l ; i, j)$, where the index pair $(k, l ; i, j)$ represents the direction of signal from $C(i, j)$ to $C(k, l)$. The coefficients $a(k, l ; i, j)$ are arranged in the feedback Template or $A$-Template. The coefficients $b(k, l ; i, j)$ are arranged in the control-Template or B-Template. The A-Template and the BTemplate are assumed to be the same for all the cells in the network. The global behavior of a $\mathrm{CNN}$ is characterized by a Template Set containing the A-Template, the B-Template, and the Bias I. The state voltage $x(i, j)$ of the cell satisfies the differential equation described by (2).

\section{1) Discrete $C N N$}

If we consider the time dependent equation 3 , the resulted discrete model for the CNN, equivalent with the continuous one, to be used in image processing is based on the following equation:

$$
\begin{gathered}
x_{i j}[n]=\sum_{C_{k l} \in N^{r}} A(i, j ; k, l) y_{k l}[n-1]+\ldots \\
+\sum_{C_{k l} \in N^{r}{ }_{i j}} B(i, j ; k, l) u_{k l}[n-1]+I \\
y_{i j}[n]=\frac{1}{2}\left(\left|x_{i j}[n]+1\right|-\left|x_{i j}[n]-1\right|\right)
\end{gathered}
$$

The performance of $\mathrm{CNN}$ is determined by the cloning template or the triple $\{\mathrm{A}, \mathrm{B}, \mathrm{I}\}$, the triplet being application dependent. In general, the cloning templates do not have to be space invariant, they can be, but it is not a necessity.

\section{2) Symmetric $C N N$}

Thus, if $\mathrm{A}(\mathrm{i}, \mathrm{j} ; \mathrm{k}, \mathrm{l})=\mathrm{A}(\mathrm{k}, \mathrm{li} ; \mathrm{i}, \mathrm{j})$, then the $\mathrm{CNN}$ is called symmetrical or reciprocal. The use of symmetric cloning templates guarantees the stability of the CNN. The use of hyperbolic periodic cloning templates guarantees the more stability, continuity and controllability of CNN.

\section{3) Asymmetric $C N N$}

In case $\mathrm{A}(\mathrm{i}, \mathrm{j} ; \mathrm{k}, \mathrm{l}) \neq \mathrm{A}(\mathrm{k}, \mathrm{l} ; \mathrm{i}, \mathrm{j})$ we are dealing with Asymmetric CNN. In spite of the stability conditions, which are difficult to be determined, the asymmetric CNN are also used in different image processing application were object orientation is needed to be taken into account while performing image processing specific transformations [14].

\section{Edge detection using CNN}

The edge detection technology is usually used to extract of edge feature of the image. Edge feature is one of the most fundamental and important feature of image. State-of-the art research proposes many theories, such as cellular neural network, genetic algorithm, wavelet transform for image processing. Considering infrared images, the brightness intensities of infrared images are representative of the temperature of object surface. Compared with rich and colorful visible images, infrared images are blurrier, have poorer resolution and clarity, and foreground/background contrast is less clear. So, in order to overcome these difficulties while dealing with infrared image, an infrared image edge detection algorithm based on the combination of the cellular neural networks (CNN) and distributed genetic algorithm (DGA) is proposed [17]. Compared with the edge detection algorithms based on cellular neural networks with template trained by particle swarm optimization, parameters search range and convergence speed are greatly improved.

\section{1) Edge detection in noisy images}

In (18), a novel a technique employing both cellular neural networks (CNNs) and linear matrix inequality (LMI) for edge detection of noisy images. The work focuses on training templates of noise reduction and edge detection CNNs. Based on the Lyapunov stability theorem, we derive a criterion for global asymptotical stability of a unique equilibrium of the noise reduction CNN. Then we design an approach to train edge detection templates, and this approach can detect the edge precisely and efficiently, i.e., by only one iteration. 
Edge detection is one of the most important popular task in image processing and pattern recognition systems. Further tasks like image segmentation, object recognition, object classification or boundary detection, are easily performed if prior edge detection is realized. However, noise is a common problem in acquisition, transmission and processing of image, which will decrease image quality. Moreover, it will lead to unexpected results when we process the images with noise by using classical edge detection operators, such as Roberts, Sobel, Prewitt and LOG operators. In (11), the authors propose a methodology employing both CNN and LMI for edge detection of noisy images. In the first step, a CNN used for reducing the noise in image waiting to be processed is designed. Then, a CNN used for detecting the edge is designed in the second step. It is shown that the templates design problem (the determination of template matrix A and B) in the two steps can be transformed into LMIs, then it is straightforward to obtain the solution by recently developed LMI Toolbox. An overall design is presented in the next figure (19).

\section{2) Edge detection in color images using CNN}

One of the difficulties in edge detection in color image is the edge definition. Indeed, in gray-level images a scalar graylevel is assigned to a pixel of image, but in color images, a color vector which consists of several components is assigned to a pixel. Another difficulty is how to integrate the contrast information contained in various components into one meaningful result. So far, monochromatic-based techniques of applying a gray-level algorithm to the single components of the image and then combining the obtained results are the most appealing edge detection methods. The work proposed in (20), focuses on using CNN for edge detection in colored images. Basically, CNN templates are determined in such manner that edge detection is performed. There are mainly two ways to design the templates: one way is to use training algorithms such as Genetic Algorithms or Linea Matrix Inequality to find desirable template; however, the templates have most possibility of over fitting the given samples and lack of generalization capability. The other way is directly according to the given task. For instance, in edge detection, we define a pixel as edge when there are more than three pixels satisfying such condition as the distance of central pixel and neighborhood greater than a threshold. However, because of complex background of color image, it is unrealistic to find a uniform threshold suitable for every component. In order to apply the aforementioned approach for edge detection, the main challenge is to integrate the three-dimensional data of color image into the CNN dynamics, because one CNN layer can only process one dimensional data. In (20), adaptive templates are employed in order to get more accurate edge in color detections. In order to be able to apply CNN to threedimensional data, a new structure of $\mathrm{CNN}$ is proposed inspired by template designing mentioned in (21), where CNN templates for hole-filling and shadow detection are reported based on gray-scale scheme. The basic control parts in CNN equation are considered scalar. This design makes threedimensional control parts in color detection be one dimensional data. In color detection, much more information than grayscale should be taken into account. Considering the change among neighboring pixels is the key aspect should be considered in edge detection, adaptive thresholds are designed based on the human vision achievement. These adaptive templates can process color image with various color and intensity information because every template carries special color and intensity character of pixel's value.

\section{3) CNN optimized by differential evolution - DE}

In (22) the authors proposed an CNN based edge detector, where the $\mathrm{A}$ and $\mathrm{B}$ matricx templates are estimated through learning algorithm, namely the differential evolution - DE. The DE algorithm is a relatively novel optimization technique for efficiently solving numerical-optimization problems. The algorithm has successfully been applied in many different problems, and gained a wide acceptance and popularity because of its simplicity, robustness, and good convergence properties. Like the genetic algorithm (GA), it employs the crossover and mutation operators and selection mechanism in order to determine the most appropriate templates which correspond to a specific task, in our case - edge detection.

As opposed to classic neural networks, the interactions within the cells in case of CNN are found only between the current cell and its neighbors. This leads to an important benefit, which is the low computational complexity, which makes them suitable for hardware implementations. The main disadvantage of satellite imagery is the need of processing large amount of data, represented by high resolution images. While benefit from their lower computational complexity, a new CNN architecture conceived for hardware implementation of complex ML-CNNs on programmable devices (19). The architecture is completely modular and expandable; all the modules share the same, properly designed, I/O interface, so the platform can be configured to accommodate CNNs of any size or structure, composed of a number of processing blocks that can be physically distributed over several FPGA boards. Taking into account advantages, they can be successfully applied on satellite images, having a short processing time due to the lower complexity of the CNN.

\section{METHODS AND SYSTEM FOR CNN BASED EDGE DETECTION}

Or automatic MRI image segmentation, the present After presenting the cellular neural networks fundamentals together with their applications in different fields such as CT image segmentation paper proposes a novel implementation for edge detection in satellite images. The proposed implementation includes a graphic user interface (GUI) and serves as a testbench in case $\mathrm{CNN}$ are used in various applications. Thus the $\mathrm{CNN}$ can be configured, while the most appropriate image preprocessing technique can be chosen, empirically, for the developed application. Moreover, the implementation offers the possibility to apply also the classic Canny filter based approach foe edge detection, which is considered to be an optimal algorithm. In this way, visual results for both cellular neural network based approach and Canny filtering can be compared in case of various satellite images. The image processing methods used within the proposed implementation for edge detection are presented in the next figure, figure 3 . The proposed methods can be classified as methods for (1) preprocessing and (2) methods for edge detection, which are detailed in the subsequent paragraphs. 
Edge detection in satellite images choose the application

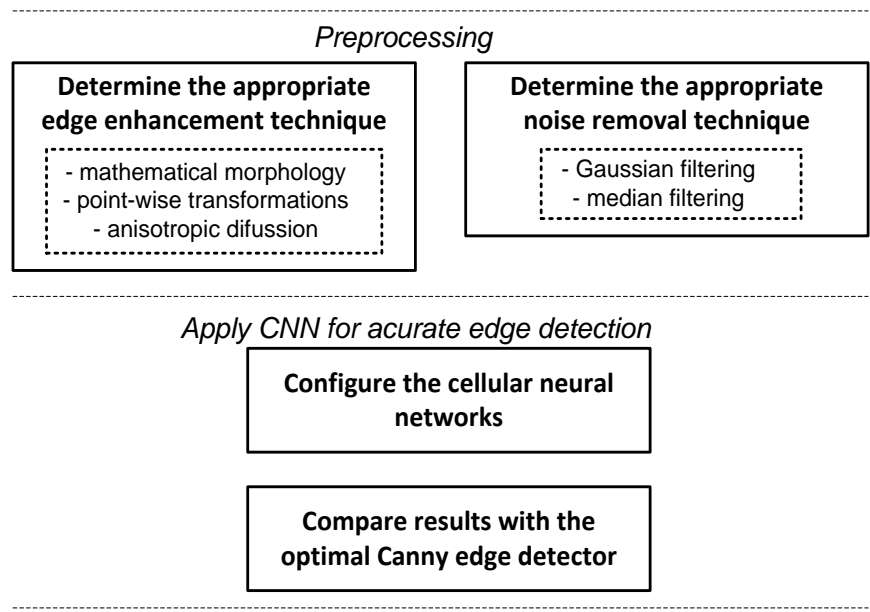

Fig. 3. Block diagram of a cell;

\section{A. Preprocessing}

The preprocessing methods are used to determine the most appropriate techniques for edge enhancement and also for noise removal. The most common techniques used for image enhancement is the spatial point-wise logarithm transformation.

In (5) a spatial logarithm transformation noted $I_{L}$ is described for a satellite image $I(x, y)$ with $\mathrm{s}(x, y)$ the current pixel and $n$ the number of bits for pixel representation.

$$
I_{L}(x, y)=\frac{\ln \left(I_{0}(x, y)+1\right)}{\ln 2^{n}} \cdot 2^{n}
$$

In (2), a novel approach based on an tangent hyperbolic transformation denoted by $I_{T}$ is proposed for image enhancement. In the second transformation $k$ determines the threshold from which the pixel intensity will be enhanced.

$$
I_{T}(x, y)= \begin{cases}\operatorname{tgh} \frac{4(I(x, y)-k)}{2^{n}-k}, & I(x, y)>=k ; \\ \operatorname{tgh} \frac{4(I(x, y)-k)}{k}, & I(x, y)<k ;\end{cases}
$$

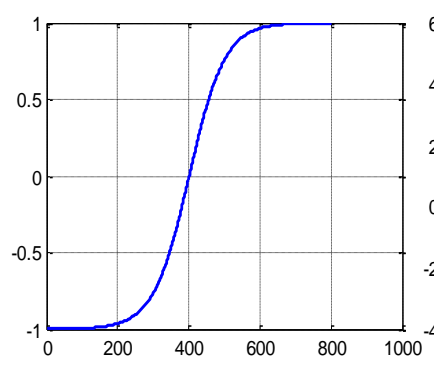

a)

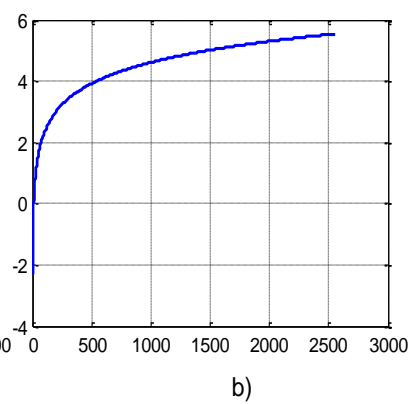

b)
Fig. 4. a) $\log$ transformation and b) arctangent hyperbolic transformation;
It is to be mentioned, after each point-wise transformation, histogram equalization is to be applied in order to fit the image in the full dynamic range. The main advantage of the arctangent hyperbolic transform is the possibility to choose the threshold from which the luminance information is increased. Under the specified threshold, $k$, the background is decreased as it can be seen in the Fig. 5.a. In this way, edge information is enhanced.

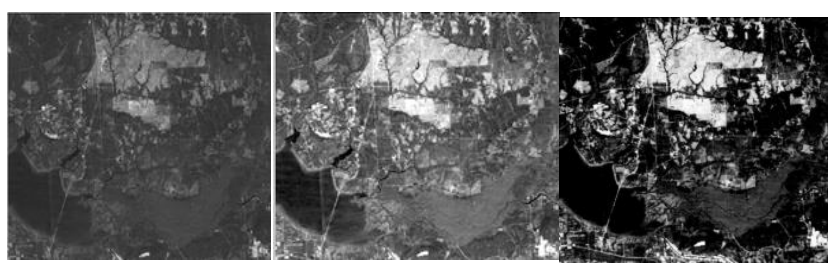

Fig. 5. a) original image - Florida, b) logarithm - transformed image, c) tatangent hyperbolic transformed image

Partial differential equations (PDEs) have various applications in image processing and computer vision. The success of these techniques is shown through their usefulness in areas such as physics and engineering sciences for a very long time. In image processing here are some of the advantages:

- They allow a reinterpretation of several classical methods such as Gaussian convolution, median filtering, dilation or erosion.

- This understanding has also led to the discovery of new methods for shape simplification, structure preserving filtering, and enhancement of different structure types.

PDE-based image processing techniques are mainly used for smoothing and restoration purposes. Typical PDE techniques for edge enhancement regard the original image as initial state of a parabolic (diffusion-like) process, and extract filtered versions from its temporal evolution.

The physical idea behind diffusion processes is presented next. The diffusion is known as a physical process that equilibrates concentration differences without creating or destroying mass. The mathematical formulation is given by the following equilibrium property:

$$
j=-D \cdot \nabla u
$$

$\mathrm{D}$ is a diffusion tensor represented by a positive symmetric matrix, which establishes the relation between the concentration gradient $\nabla u$ and a flux $j$ which aims to compensate for this gradient. In case $j$ and $\nabla u$ are parallel, the diffusion is called isotropic. The property of the diffusion of not to destroy mass/information is expressed by the continuity equation (8).

$$
\partial_{t} u=-\operatorname{div} j
$$

Considering all of the above, the diffusion equation is given by equation (9), which appears in case of different transport processes. Regarding the diffusion tensor, if it depends on the 
evolving image in time domain, the diffusion is called nonlinear.

$$
\partial_{t} u=\operatorname{div}(D \cdot \nabla u)
$$

If the diffusion tensor is constant over the whole image domain, one speaks of homogeneous or isotropic diffusion, and a space-dependent filtering is called inhomogeneous or anisotropic.

\section{1) Anisotropic diffusion for edge enhancement}

Perona and Malik (5) propose a nonlinear diffusion method for avoiding the blurring and localization problems, by applying an inhomogeneous process that reduces the diffusivity at those locations which have a larger likelihood to be edges. The probability for a specific area to be edge is denoted by $|\nabla u|^{2}$. The Perona-Malik equation is (10).

$$
\begin{aligned}
& \partial_{t} u=\operatorname{div}\left(g\left(|\nabla \mathrm{u}|^{2}\right) \cdot \nabla u\right) \\
& g\left(s^{2}\right)=\frac{1}{1+s^{2} \lambda^{2}}
\end{aligned}
$$

The experiments of Perona and Malik were visually very impressive: edges remained stable over a very long time. It was demonstrated that edge detection based on this process clearly outperforms the linear Canny edge detector, even without applying non-maxima suppression and hysteresis thresholding. This is due to the fact that diffusion and edge detection interact in one single process instead of being treated as two independent processes which are to be applied subsequently. Considering this advantage, the user of our proposed graphic user interface for edge detection of satellite images will have the opportunity to choose this preprocessing technique before edge detection.

Results of the conventional anisotropic diffusion (Perona \& Malik) upon a gray scale image aiming edge enhancement are presented next. A 2D network structure of 8 neighboring nodes is considered for diffusion conduction. The parameters to be chosen for the diffusion are the number of iterations Num_Iter, integration constant Delta_T which is set usually to maximum value and the gradient modulus threshold that controls the conduction denoted by Kappa.

Moreover, the conduction coefficient functions proposed by Perona and Malik are given by the following equation, eq. (12) and (13).

$$
g\left(s^{2}\right)=e^{-s^{2} \lambda^{2}}
$$

In case of eq. 12, high-contrast edges are privileged over low-contrast ones, while in case of eq. (13), wide regions are privileged over the smaller ones.

$$
g\left(s^{2}\right)=\frac{1}{1+s^{2} \lambda^{2}}(13)
$$

Further on, some examples of how the filtering is performed will be presented considering different parameter setups. First, in figure 6.a, diffusion is applied using the conduction coefficient from eq. 13, with different numbers of iteration, and different values for the Kappa threshold.

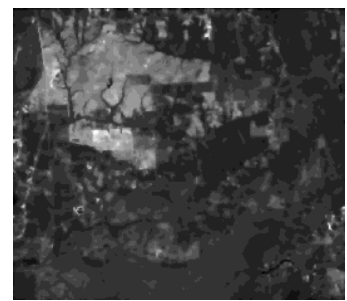

a)

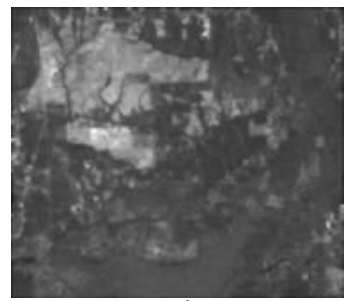

c)

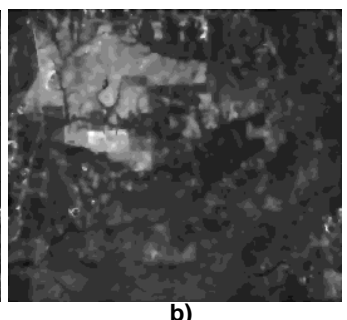

b)

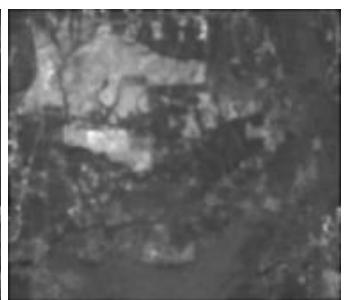

d)
Fig. 6. Anisotropic diffusion applied for edge enhancement in case of original image Florida from figure 5, considering the diffusion function from eq. (2): a) Num_iter $=25$, Kappa $=10$, b) Num_iter $=15$, Kappa $=30$, c) Num_iter $=15$, Kappa $=30$, d) Num_iter $=25$, Kappa $=30$.

As it can be observed in the previous figure, the parameter setup for edge enhancement can be determined empirically. In our case, using a gradient threshold of 30 generates an intense diffusion which damages the edges. The optimal parameters are Kappa $=10$ and the number of iteration Num_iter $=15$, which accurately preserves the edges.

\section{2) CNN for edge detection}

The main principle used in cellular neural networks is that the satellite image evolves in time, and converges to an image were the edges are visible. Thus, cellular neural networks are governed by a differential equation, which is given by eq. (3) and (4). The interpretation of the previous equation is as follows: for each step ( $t$ to $t+1)$ in applying the cellular neural network, the element $x_{i j}$ is replaced by a combination of the previous $x_{i j}$, $I_{\text {bias }}$ and $\left(a_{k} x_{i j}+b_{k} u_{k}\right)$ were $a_{k}$ and $b_{k}$ are called the control matrices. For a more detailed view on how CNN work, in the next figure, figure 7, a block diagram is presented.

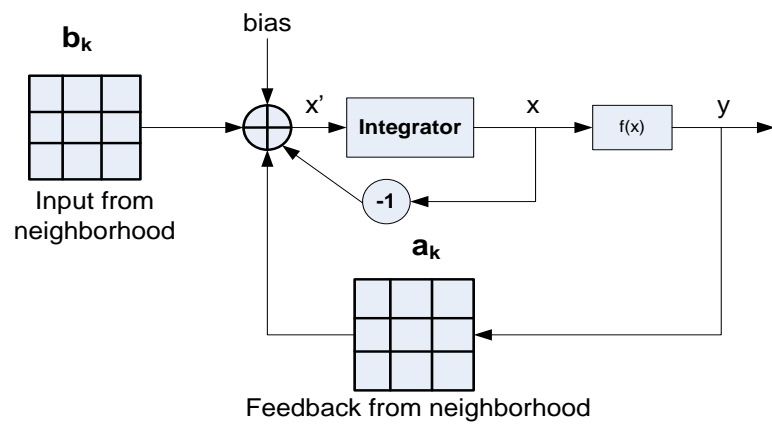

Fig. 7. Block scheme of CNN implementation 
The proposed image processing techniques for edge detection using CNN were applied on the San Francisco bridge satellite image only for exemplification. The effect of the Perona and Malik anisotropic diffusion on edge detection is marked in the figure 7.

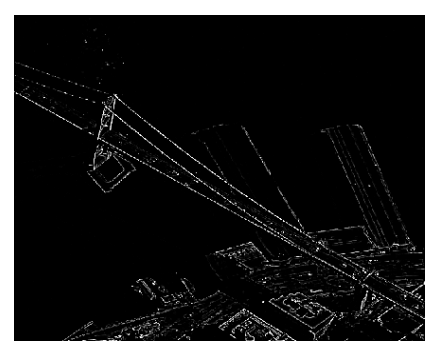

a) Cellular neural network with anisotropic difussion applied

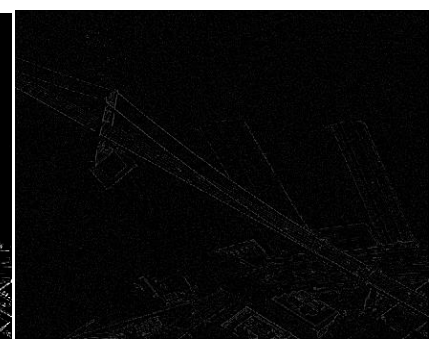

b) Cellular neural network without preprocessing techniques (anisotropic difussion)
Fig. 8. Preliminary results of the CNN and edge enhancement techniques on San Francisco ;

Thus, the edges are more accurate detected if the preprocessing techniques are used Fig. 7.a compared with the situation when no preprocessing technique is used Fig 7.b.

Further on, the implementation and the experimental results of $\mathrm{CNN}$ based edge detection used in satellite image processing with application in geology are presented. The nature of the aforementioned images demands a special parameterization of PDE filters (Perona and Malik) and of the preprocessing methods. All steps performed by our image processing systems and the corresponding results are described further on.

\section{EXPERIMENTAL RESULTS}

The subsequent paragraphs present the way our proposed system is used to process satellite images with application in geophysics. A set of three satellite images of Egypt areas are chosen for processing: the first image is called "El dist", the second one "G hammad" and the third one is called "Elhoufof". The nature of the aforementioned images demands a special parameterization of PDE filters (Perona and Malik) and of the preprocessing methods. The images containing the edges resulted after applying our image processing system are presented.

\section{A. Proposed system for CNN edge detection}

The proposed system applies edge detection on grayscale images, thus in case of color image a conversion from RGB to Gray-Scale is performed.

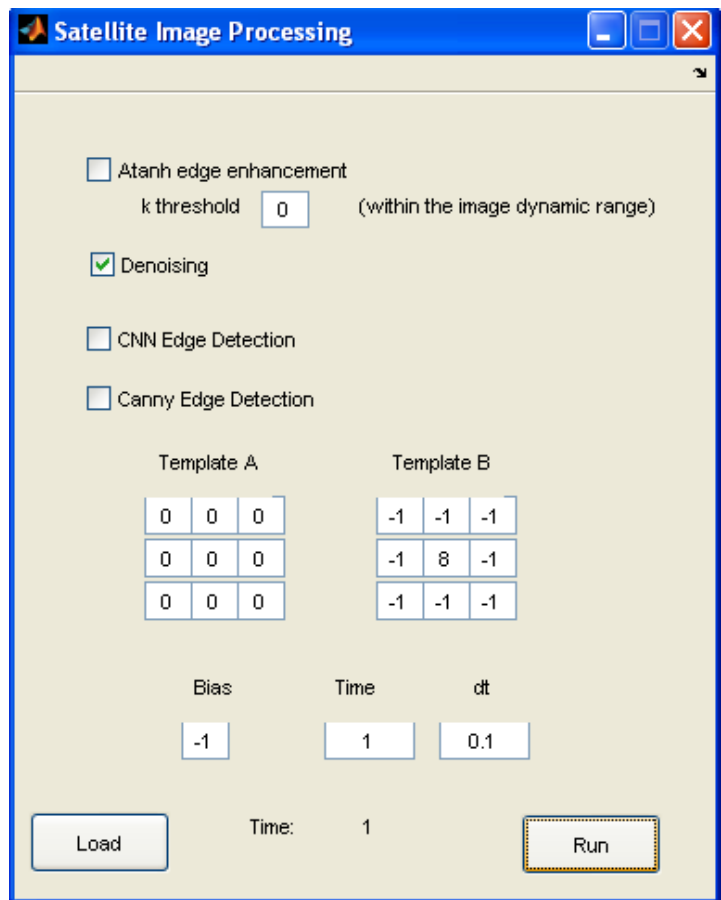

Fig. 9. Block scheme of CNN implementation

An graphic user interface is also created in order to create an interactive environment for the user to process satellite images Different preprocessing methods can be chosen by the user for edge enhancement or noise removal. Arctangent hyperbolic transform is used to enhance the edges within the image. The arctangent hyperbolic is based on a threshold $k \_$threshold, selected by the user from the original image. The threshold is equal to the pixel intensities where the edges of interest are found. In case of different applications, different edges are wanted to be detected. Thus our application is focused on the user needs.

Moreover, the user has the option to apply Perona and Malik denoising techniques. The main advantage is the edge preserving feature of this filter and also edge enhancement.

The configuration of the CNN can be specified by the Template $A$ and Template $B$ matrices and by the Bias, Time and $d t$ parameters. After configuring the $\mathrm{CNN}$ network and choosing the preprocessing techniques, the edge detection can be performed. As ground truth for the edge detection the optimal Canny filter can also be applied on the satellite images. 


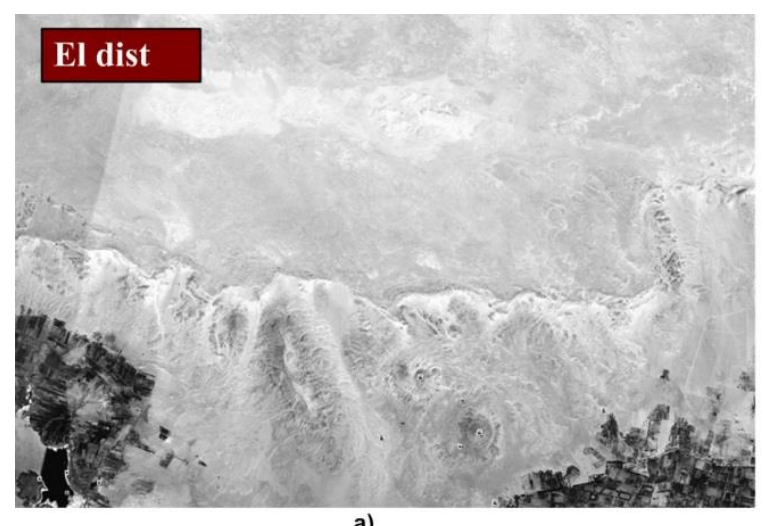

a)

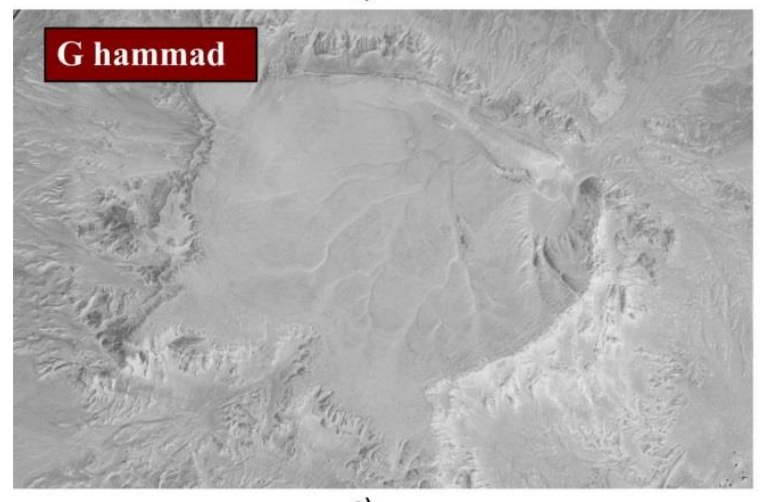

c)

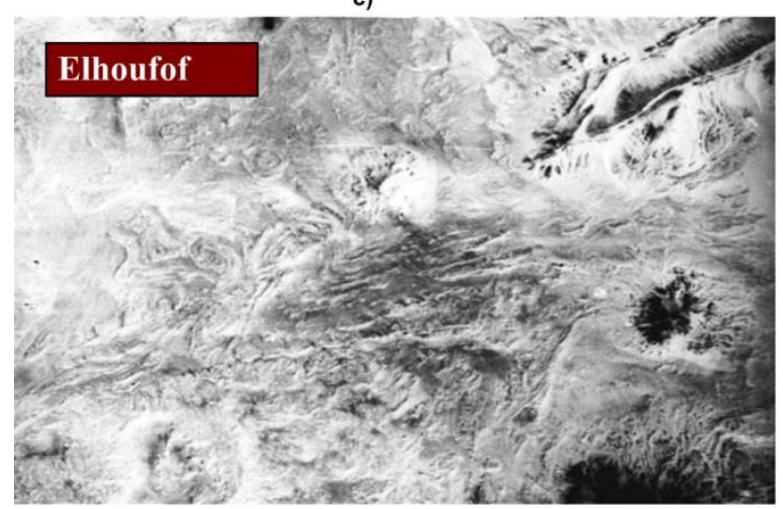

e)

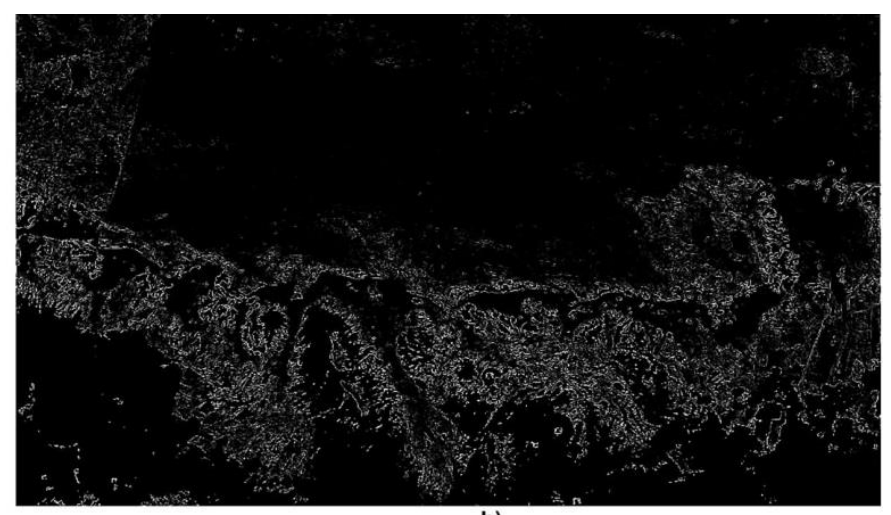

b)
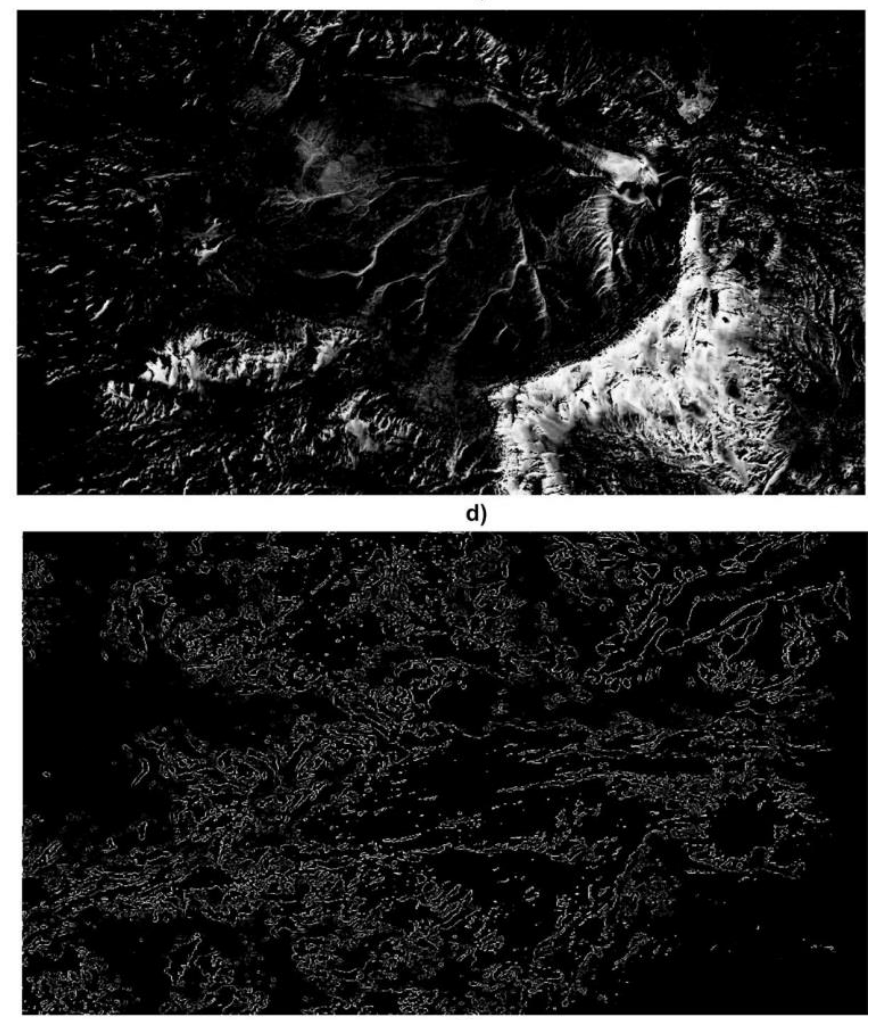

f)

Fig. 10. The input satellite images on the left column and the images with edge detected on the right column

\section{B. Parameters setup}

In order to process satellite images from Egypt used in geophysics applications, we set up our proposed system to match the image features. Thus, firstly the $k$ threshold was interactively selected for each microarray image; the values for each image are presented in Table 1. Anisotropic diffusion applied for edge enhancement and noise removal is also parameterized. Considering the details found within the images, an increased number of iterations and an increased $k$ factor are considered. Thus a number of 20 iterations are chosen, whereas a kappa factor of 15 are chosen.

Various template matrices $a_{k}$ and $b_{k}$ can be used for edge detection and other various task in image processing. For edge detection, the content for the $a_{k}$ and $b_{k}$ template matrices is presented by eq. 12 . The initial state is considered the original image, which evolves in time in such manner that the edge will be obtain in the end. Moreover, the $I_{\text {bias }}$ is considered to be -1 . The resulted edges are presented in Figure 10.

$$
a_{k}=\left(\begin{array}{lll}
0 & 0 & 0 \\
0 & 1 & 0 \\
0 & 0 & 0
\end{array}\right), b_{k}=\left(\begin{array}{ccc}
-1 & -1 & -1 \\
-1 & 8 & -1 \\
-1 & -1 & -1
\end{array}\right) \text { and } I_{\text {bias }}=-1
$$

\section{Accuracy comparison}

Further on, we will define a new quality metric in order to estimate the number of pixels, denoted by $p_{i j}$, involved in defining the edge. Thus let us consider the set denoted by the set $e=$ \{number of $p_{i j} / p_{i j}$ belonging to the edge $\}$. The set $e_{C N N}$ will be computed for the edges determined by the CNN method and also the set $e_{\text {Canny }}$ will be computed for the edges 
determined with the canny filtering. The difference will show the difference between the two methods with regards to the accuracy of the results. We will consider the edges detected in the satellite images from Table 1 using the both image processing methods, CNN and Canny respectively. The sets $e$ will be computed for both images in case of both approaches for edge detection.

TABLE I. BENCHMARK RESULTS OF THE CASCADE OSCILLATORS MODEL

\begin{tabular}{|l|c|c|c|}
\hline \multicolumn{1}{|c|}{ Satellite image } & El dist & G hammad & Elhoufhof \\
\hline Image size & $1877 \times 1255$ & $3129 \times 118$ & $1882 \times 1400$ \\
\hline dpi (vertical and horizontal) & $300 \times 300$ & $300 \times 300$ & $300 \times 300$ \\
\hline k_threshold & 180 & 245 & 210 \\
\hline Number of edge pixels $\mathbf{e}_{\mathrm{CNN}}$ & $185 \mathrm{k}$ & $242 \mathrm{k}$ & $146 \mathrm{k}$ \\
\hline Number of edge pixels $\mathbf{e}_{\text {Canny }}$ & $37 \mathrm{k}$ & $45 \mathrm{k}$ & $42 \mathrm{k}$ \\
\hline
\end{tabular}

As the Table 1 presents, the edges detected by the proposed approach are composed of an increased number of pixels as compared with the Canny edge detector edges. This means, in case of images were a lot of details are included (as satellite images are) the proposed method delivers good results, even more detailed results than existing approaches for edge detection.

\section{CONCLUSIONS}

Edge detection is an image processing task used in various types of image processing applications. In case of satellite image processing, edge detection it is of high importance due to the fact it detects different areas of interest for different applications. Thus, in the field of meteorology, detects areas on thermograph images which correspond to different temperatures or as another example, in the field of agriculture edge detection marks areas were different kind of crops are planted. Considering the importance of edge detection, we propose an image processing workflow which includes a cellular neural network based. The proposed image processing workflow delivers accurate results with regards to the edges within the satellite images. In order to prove the improved results of our approach based on CNN used in conjunction with the appropriate image preprocessing technique, we compared the obtained results with the classic edge detection techniques as Canny edge detector filter. The quality measure used for comparison is the quantity of pixels which belong to the edges. Considering the quality metric mentioned before it has been proved that our image processing chain delivers edge detection more accurate. Thus the edges determined by our method are composed of more pixels than Canny edge detector.

To conclude, the main benefits of our work for edge detection in case of satellite images are presented. An image processing platform was built were the user has the possibility to chose the image processing methods to be used for edge detection and their specific parameters. Thus, arctangent hyperbolic transform, together with anisotropic diffusion and cellular neural network parameters can be used.
It is to be mentioned that satellite images are of high resolutions and contain a lot of information. Our proposed methods is adapted to the characteristics of satellite images and the detected edges are composed of an increased number of pixels, preserving salient information in a better way than classic image processing techniques as Canny edge detector.

\section{FUTURE WORK}

The proposed system for edge detection offers the user the possibility to choose different preprocessing techniques and two edge detection algorithms: CNN and Canny filter. Due to the fact that the image processing system is easy to use, it can be applied in various image processing applications. Thus, three main directions are distinguished as part of the future work to be developed using the proposed system for edge detection.

First objective of our future work is to use the proposed edge detection algorithm in agricultural application which makes use of satellite imagery. Thus, the edge detection algorithm is used to determined different areas on earth were different crops are seeded. Thus, by detecting the edges and consequently each area, the crops can be monitored and the national authorities in agriculture have a report on how each different crop evolved.

The second objective of future work is to use the edge detection system in meteorological application in order to detect different areas on satellite images affected by different meteorological phenomena.

\section{REFERENCES}

[1] Landsat: A Global Land-Observing Program, Fact Sheet 023-03 (March 2003)

[2] Liang, S. 2000. "Narrowband to broadband conversions of land surface albedo I algorithms." Remote Sensing of Environment 76, 213-238

[3] Campbell, J. B. 2002. Introduction to Remote Sensing. New York London: The Guilford Press

[4] http://en.wikipedia.org/wiki/Satellite_imagery

[5] P. Perona, J. Malik, Scale space and edge detection using anisotropic diffusion, IEEE Trans. Pattern Anal. Mach. Intell., Vol. 12, 629-639, 1990

[6] P. Soille, Morphological Image Analysis, Springer-Verlag, 2003.

[7] Tanvir A. Abassi, Usaid Abassim A Proposed FPGA Based Architecture for Sobel Edge Detection Operator, Journal of Active and Passive Electronic Devices, pp. 271-277, 2007

[8] Chua, L.O. and Yang, L. "Cellular Neural Networks: Theory and Applications", IEEE Trans. on Circuits and Systems, (CAS), Vol.35 (1988), 1257-1290

[9] Roska, T. and Vandewalle, J. "Cellular Neural Networks". (John Wiley\&Sons), (1993)

[10] Pham Hong Long, Pham Thuong Cat "Real-time Image Processing by Cellular Neural Network Using Reaction-Diffusion Model", International Conference on Knowledge and Systems Engineering, pp. $93-99,2009$

[11] Te-Jen Su, Cian-Pin Wei, Shih-Chun Huang, Chia-Ling Hou, "Image noise cancellation using linear matrix inequality and cellular neural network" Optics Communications, 281, 23, pp. 5706-5712

[12] P.Elango a and K.Murugesan, "Digital Image Inpainting Using Cellular Neural Network," Int. J. Open Problems Compt. Math., Vol. 2, No. 3, 2009

[13] Chua, L.O. and Roska, T. „The CNN Paradigm. IEEE Transactions on Circuits and Systems (Part I)“, CAS-40, 3 (1993), 147-156 
[14] See Henry J. Gomez, An Agent of Change the Web Has Transformed Today's Real Estate Industry, THE PLAIN DEALER (Cleveland), Mar. 19, 2007

[15] Wang Shitong, Fu Duana, Xu Mina, Hu Dewenc, "Advanced fuzzy cellular neural network: Application to CT liver images," Artificial Intelligence in Medicine 39, pp. 65-77, 2007

[16] Antonio Cerasaa et al., "A Cellular Neural Network methodology for the automated segmentation of multiple sclerosis lesions," Journal of Neuroscience Methods, 203, 193-199, 2012

[17] Wei Wang, Li-Jun Yang, Yu-Ting Xie, You-wei, "Edge detection of infrared image with CNN DGA algorithm," Optik 125 (2014) 464467.

[18] Huaqing Li, Xiaofeng Liao, Chuandong Li, Hongyu Huang, Chaojie $\mathrm{Li}$, "Edge detection of noisy images based on cellular neural networks" Commun Nonlinear Sci Numer Simulat 16, 3746-3759, 2011

[19] J. Javier Martınez, Javier Garrigo, Javier Toledo, Manuel Fernandez, "An efficient and expandable hardware implementation of multilayer cellular neural networks", Neurocomputing, 114, pp. 54-62, 2013

[20] S. Deng et al., "Application of new advanced CNN structure with adaptive thresholds to color edge detection", /Commun Nonlinear Sci Numer Simulat 17 (2012) 1637-1648

[21] Matsumoto T, Chua LO, Furukawa R. CNN cloning template: holefiller. IEEE Trans Circuits Syst 1990;37(5):635-8.

[22] Alper Basturk, Enis Gunay, "Efficient edge detection in digital images using a cellular neural network optimized by differential evolution algorithm", Expert systems with applications, 36, pp. 2645 $2650,2009$. 